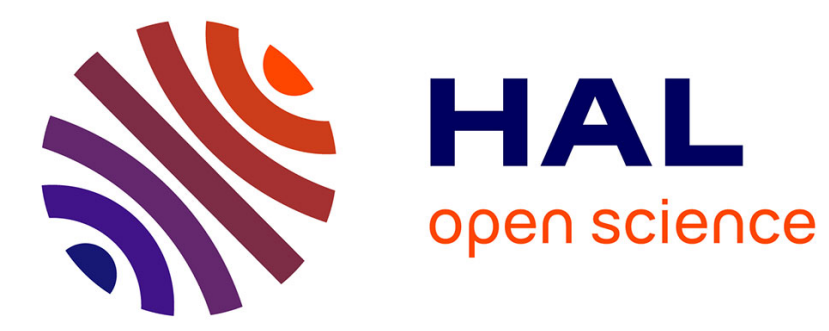

\title{
Approche dynamique et intégrée d'un territoire forestier dans les Pyrénées catalanes.
}

Hélène Chevallier, Cathy Bassignot, Jérôme Bussière, Bruno Mariton

\section{To cite this version:}

Hélène Chevallier, Cathy Bassignot, Jérôme Bussière, Bruno Mariton. Approche dynamique et intégrée d'un territoire forestier dans les Pyrénées catalanes.. Revue forestière française, 2001, 53 (sp), pp.226-234. 10.4267/2042/5300 . hal-03443516

\section{HAL Id: hal-03443516 https://hal.science/hal-03443516}

Submitted on 23 Nov 2021

HAL is a multi-disciplinary open access archive for the deposit and dissemination of scientific research documents, whether they are published or not. The documents may come from teaching and research institutions in France or abroad, or from public or private research centers.
L'archive ouverte pluridisciplinaire HAL, est destinée au dépôt et à la diffusion de documents scientifiques de niveau recherche, publiés ou non, émanant des établissements d'enseignement et de recherche français ou étrangers, des laboratoires publics ou privés. 


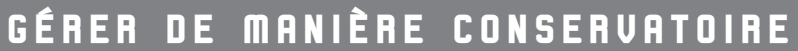

\section{APPROCHE DYNAMIQUE ET INTÉGRÉE D'UN TERRITOIRE FORESTIER dans les Pyrénées catalanes}

Hélène Chevallier - Cathy Bassignot - Jérôme Bussière - Bruno Mariton

Patrimoine naturel forestier et paysage sont au cœur des réflexions menées actuellement autour d'un projet de parc naturel régional dans les Pyrénées catalanes. En effet, la forêt s'y étend sur les étages supraméditerranéen à subalpin, offrant une grande diversité de milieux et de paysages.

Parallèlement, cette couverture forestière engendre une abondante ressource : accompagner la filière-bois fait donc naturellement partie des objectifs du projet de parc naturel régional.

Par ailleurs, comme dans beaucoup de territoires ruraux, la déprise et l'évolution des techniques agricoles ont pour conséquence une progression de cette couverture forestière dont dépendent aujourd'hui la qualité des paysages et la vocation des terres.

La forêt représente donc un enjeu pour ce territoire. Dans le cadre du projet de parc naturel régional, deux actions importantes sont actuellement menées, visant à une meilleure connaissance de ces forêts et à une approche la plus complète possible pour préparer une gestion adaptée.

\section{UN TERRITOIRE, UN PATRIMOINE FORESTIER, DES FORÊTS... ET DES HOMMES}

\section{Brève présentation forestière du territoire}

Le projet de Parc naturel régional des Pyrénées catalanes concerne 57 communes, pour une surface de près de 130000 ha, boisée à $45 \%$. Ce territoire est découpé en trois grandes entités naturelles principales : le plateau de Cerdagne, le plateau du Capcir et le Haut-Conflent (moyenne vallée de la Têt, qui relie les hauts-plateaux à la plaine littorale).

- Les forêts du Capcir sont présentes essentiellement sur les versants entourant la dépression du Capcir, seule la forêt de la Matte occupe le plateau. Elles couvrent $60 \%$ de la surface. Les peuplements sont composés essentiellement de Pin à crochets ou de Pin sylvestre, en fonction notamment de l'exposition et de l'altitude. Un peu plus de $20 \%$ des massifs boisés sont des propriétés privées. De faible surface (96\% sont inférieures à 4 ha, avec une moyenne de 0,3 ha par propriété), celles-ci occupent généralement les bordures de forêts communales ou domaniales, suite au recul de l'activité agricole.

- Le taux de boisement de la Cerdagne est de $24 \%$. Là encore, les forêts occupent essentiellement les versants. Elles sont communales. Il s'agit de peuplements de Pin à crochets, en mélange parfois avec le Pin sylvestre. Le plateau reste peu boisé. Les propriétés privées sont cantonnées à proximité des villages ou en bordure des massifs communaux, sur les parcelles délaissées par 


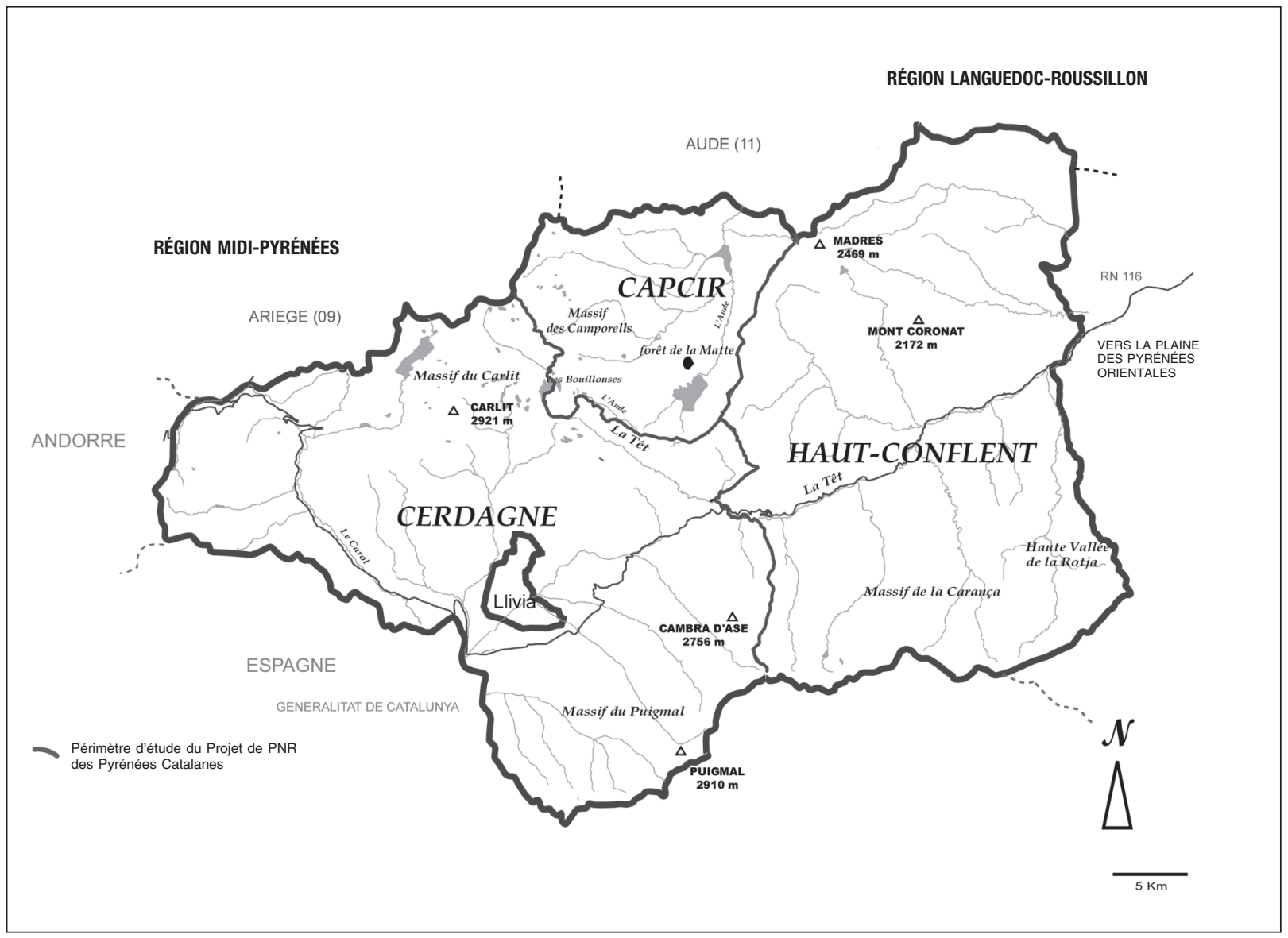

les pratiques agricoles. Elles se présentent alors sous forme d'accrus ou de plantations. Les bordures de chemins ruraux et les haies bocagères encore bien présentes dans le paysage renforcent la part des feuillus (Frêne, Noisetier notamment).

- Dans le Haut-Conflent boisé à environ $60 \%{ }^{(1)}$, on retrouve en altitude le Pin sylvestre et le Pin à crochets, avec le Hêtre et le Sapin de façon plus dispersée et, sur les zones plus basses, les principales essences composant les peuplements sont le Chêne vert, le Chêne pubescent, le Frêne ainsi que les Pins sylvestre et noir sur les zones les plus pauvres. 60 \% des forêts sont privées. La gestion y est très hétérogène, dépendante des conditions stationnelles plus ou moins favorables, de la surface concernée, de la desserte existante ou non...

Par ailleurs, la forte dynamique forestière naturelle sur les espaces en déprise est très marquée, sur l'ensemble du territoire.

(1) Il s'agit d'une estimation, le Haut-Conflent étant inclus dans la région IFN “Conflent, boisée $\simeq 50 \%$ " (données IFN). 
D'un point de vue patrimonial, les inventaires ont recensé 4 grands habitats prioritaires, au sens de la Directive européenne ${ }^{(2)}$ :

- forêts de Pin à crochets sur calcaire $\left(42.4^{(3)} ; 9430^{(4)}\right)$,

- ripisylves dont galeries d'Aulnes (44.3; 91Eo),

- forêts de Pin de Salzmann (42.63; 9533),

- forêts de ravins $(41.4 ; 9180)$.

La gestion patrimoniale des principaux habitats forestiers présents pose un certain nombre de questions telles que celles du tableau I.

TABLEAU I Questions posées par la gestion patrimoniale des principaux habitats forestiers

\begin{tabular}{|c|c|}
\hline Grand type forestier & "Problématique de gestion" \\
\hline Pineraie à crochets & $\begin{array}{l}\text { - hiérarchisation des différents faciès forestiers et impact sur la gestion: diver- } \\
\text { sification des essences } \\
\text { - étude et valorisation du bois du Pin à crochets } \\
\text { - problème sanitaire lié à l'armillaire } \\
\text { - habitat d'espèce: Grand Tétras (Tetrao urogallus) }\end{array}$ \\
\hline Pineraie Pin sylvestre & $\begin{array}{l}\text { - grand intérêt de certaines localités (forêt de la Matte) } \\
\text { - problème phytosanitaire: chenille processionnaire } \\
\text { - habitat d'espèce: Isabelle (Actias graellsia, lépidoptère) }\end{array}$ \\
\hline $\begin{array}{l}\text { Sapin } \\
\text { Hêtraies-sapinières }\end{array}$ & - où favoriser le Sapin? \\
\hline $\begin{array}{l}\text { Châtaigneraie, chênaie, } \\
\text { hêtraie... } \\
\text { en Haut-Conflent }\end{array}$ & $\begin{array}{l}\text { - valorisation de l'existant } \\
\text { - orientations et diversification: exemple feuillus précieux sur terrasse, } \\
\text { agrosylvopastoralisme }\end{array}$ \\
\hline Pin de Salzmann & - protection et conservation: originalité génétique? intérêt en RTM? \\
\hline Ripisylves & $\begin{array}{l}\text { - meilleure connaissance du fonctionnement } \\
\text { - gestion du cours d'eau }\end{array}$ \\
\hline
\end{tabular}

\section{Objectifs et orientations : regards sur les premiers éléments de rédaction de la charte}

Tant par leur patrimoine naturel que paysager ou culturel, les Pyrénées catalanes sont un territoire d'exception. Le projet de création d'un Parc naturel régional contribue à structurer la volonté de développement durable ressentie par les acteurs locaux.

Six grands objectifs sont ressortis des premières réflexions menées depuis 1996 pour la structure de gestion de ce Parc naturel régional :

« - contribuer à la préservation, la gestion et la valorisation du patrimoine diversifié, original, et fragile,

- contribuer au développement harmonieux et durable, dans le respect de la diversité de ce territoire montagnard vivant et contrasté,

(2) Directive 92/43/CEE du 21 mai 1992 dite "Directive Habitats".

(3) Code CORINE Biotopes.

(4) Directive "Habitats" : code Natura 2000. 
- soutenir l'expression de l'important potentiel de développement du territoire au dynamisme économique contrasté,

- soutenir la construction des solidarités nécessaires à la mise en cohérence des initiatives locales,

- affirmer l'image forte et cohérente du territoire,

- assurer la redécouverte du territoire à destination des acteurs locaux et des visiteurs " (5).

La forêt étant un des éléments fondateurs du patrimoine naturel, paysager et économique de ce territoire, nombre des orientations stratégiques ont pour objectif sa valorisation, sous diverses formes.

Dans le cadre de ce parc naturel régional, un des rôles premiers resterait l'animation et l'assistance technique auprès des acteurs forestiers du territoire autour de grands objectifs.

Nous proposons, dans le tableau II (p. 230), quelques exemples d'orientations forestières (5).

Ainsi, dans ce contexte, gestionnaires forestiers, éleveurs, agriculteurs, naturalistes et scientifiques se sont retrouvés dans le cadre du projet de parc naturel régional des Pyrénées catalanes autour de différents projets. Regroupés en comités techniques, ils travaillent à l'élaboration d'un diagnostic le plus fin possible du territoire, ainsi qu'à la réalisation d'actions concrètes. Au-delà de la création du parc naturel régional, l'objectif est de faire émerger une cohérence de territoire, une meilleure valorisation de ses ressources, qu'elles soient naturelles, touristiques, culturelles ou économiques.

Nous présentons ici deux directions de travail actuelles sur le pôle de la forêt, relatives au regroupement, tout d'abord, à l'information et à la communication, ensuite.

\section{REGROUPER POUR POUVOIR GÉRER}

Comme dans beaucoup d'espaces ruraux, l'éclatement des propriétaires, en nombre et dans l'espace, rend difficile une gestion efficace. Celle-ci ne peut pas s'envisager sur des microparcelles. Un regroupement est nécessaire pour former des entités plus homogènes et gérables.

Se regrouper pour mieux fonctionner n'est pas nouveau. II nous paraît cependant intéressant de revenir sur le contexte et les opérations effectuées sur les Pyrénées catalanes.

Dans le cadre d'une opération de préfiguration du parc naturel régional des Pyrénées catalanes, un périmètre comprenant des parcelles privées et communales a servi de support à un diagnostic multivarié. Cette action a été menée sur un secteur de la forêt de la Matte : 7 ha en forêt communale ( 2 parcelles forestières) et environ 8 ha en forêt privée (plus de 100 parcelles).

Divers analyses et inventaires ont été effectués : inventaire botanique, étude avifaunistique, description sylvicole et sanitaire des peuplements, diagnostics pastoral et paysager, mise à plat des enjeux touristiques.

Trois grands types de parcelles ont été décrits :

1- parcelles, récemment encore, agricoles, colonisées par de la régénération naturelle jeune (moins de 15 ans),

(5) Pré-Charte, Projet de Parc naturel régional des Pyrénées catalanes. Agence méditerranéenne de l’Environnement. Document de travail. Juillet 2000. 
2- parcelles autrefois agricoles, avec régénération naturelle ou plantation (entre 15 et 30 ans d’âge) (cf. photo 1, p. 231),

3- peuplements naturels de Pin sylvestre (plus de 30 ans).

TABLEAU II Quelques orientations forestières pour le futur parc naturel régional

\begin{tabular}{|c|c|c|c|}
\hline \multicolumn{3}{|c|}{ Grandes orientations } & \multirow{2}{*}{$\begin{array}{l}\text { Exemples d'objectifs validés } \\
\text { - Promouvoir des techniques } \\
\text { complémentaires respectueuses } \\
\text { de l'environnement (traction } \\
\text { animale, scie mobile...) }\end{array}$} \\
\hline \multirow[t]{4}{*}{$\begin{array}{l}\text { Patrimoine } \\
\text { et territoire: } \\
\text { préserver, gérer } \\
\text { et valoriser } \\
\text { le patrimoine }\end{array}$} & \multirow[t]{2}{*}{$\begin{array}{l}\text { Milieux et ressources } \\
\text { naturelles }\end{array}$} & $\begin{array}{l}\text { Qualité } \\
\text { de l'environnement }\end{array}$ & \\
\hline & & $\begin{array}{l}\text { Favoriser une gestion } \\
\text { concertée }\end{array}$ & $\begin{array}{l}\text { - Action de préfiguration: amé- } \\
\text { nagement concerté } \\
\text { sur un secteur de la forêt } \\
\text { de la Matte (Capcir) }\end{array}$ \\
\hline & \multirow[t]{2}{*}{ Paysage } & Qualité des paysages & $\begin{array}{l}\text { - Aider à préserver la forêt } \\
\text { et les parcelles agricoles } \\
\text { d’éventuelles dégradations } \\
\text { (feux, fréquentation, } \\
\text { problèmes sanitaires...) }\end{array}$ \\
\hline & & $\begin{array}{l}\text { Assurer la pérennité } \\
\text { des paysages ruraux } \\
\text { et des milieux naturels } \\
\text { directement issus } \\
\text { des pratiques agricoles } \\
\text { et forestières actuelles } \\
\text { et passées }\end{array}$ & $\begin{array}{l}\text { - Prendre en compte l’intérêt } \\
\text { des arbres, bosquets et haies } \\
\text { - Promouvoir un équilibre } \\
\text { agro-sylvo-pastoral }\end{array}$ \\
\hline \multirow{4}{*}{$\begin{array}{l}\text { Économie } \\
\text { et coopération: } \\
\text { promouvoir le Parc } \\
\text { naturel régional } \\
\text { comme un espace } \\
\text { économique } \\
\text { de qualité }\end{array}$} & $\begin{array}{l}\text { Promouvoir une } \\
\text { activité commerciale } \\
\text { et artisanale }\end{array}$ & $\begin{array}{l}\text { Allier performance } \\
\text { économique } \\
\text { et environnementale }\end{array}$ & $\begin{array}{l}\text { - Énergies renouvelables } \\
\text { (énergie-bois) }\end{array}$ \\
\hline & \multirow[t]{3}{*}{$\begin{array}{l}\text { Dynamiser la vie } \\
\text { économique autour } \\
\text { de l'agriculture } \\
\text { et de l'exploitation } \\
\text { forestière }\end{array}$} & $\begin{array}{l}\text { Valoriser l'image } \\
\text { des productions locales } \\
\text { et accroître leur qualité }\end{array}$ & $\begin{array}{l}\text { - Sensibiliser les propriétaires } \\
\text { forestiers à la nécessité } \\
\text { de faire des travaux } \\
\text { et d'entretenir leurs forêts } \\
\text { - Communiquer sur les qualités } \\
\text { technologiques des essences } \\
\text { forestières (notamment le Pin } \\
\text { à crochets) }\end{array}$ \\
\hline & & $\begin{array}{l}\text { Améliorer l'utilisation } \\
\text { et la valorisation } \\
\text { des ressources locales }\end{array}$ & $\begin{array}{l}\text { - Accompagner } \\
\text { le développement des filières } \\
\text { locales de transformation: } \\
\text { mise en œuvre du bois } \\
\text { - Préconiser l'utilisation des } \\
\text { essences locales en favorisant } \\
\text { le rapprochement des actifs } \\
\text { de la filière-bois et la mise } \\
\text { en adéquation de l'offre } \\
\text { et de la demande }\end{array}$ \\
\hline & & $\begin{array}{l}\text { Accompagner } \\
\text { la diversification } \\
\text { des produits et services }\end{array}$ & $\begin{array}{l}\text { - Développer la réflexion } \\
\text { sur la production d'espèces } \\
\text { forestières variées } \\
\text { - Promouvoir les techniques } \\
\text { complémentaires (débardage } \\
\text { à cheval, scie mobile...) }\end{array}$ \\
\hline
\end{tabular}




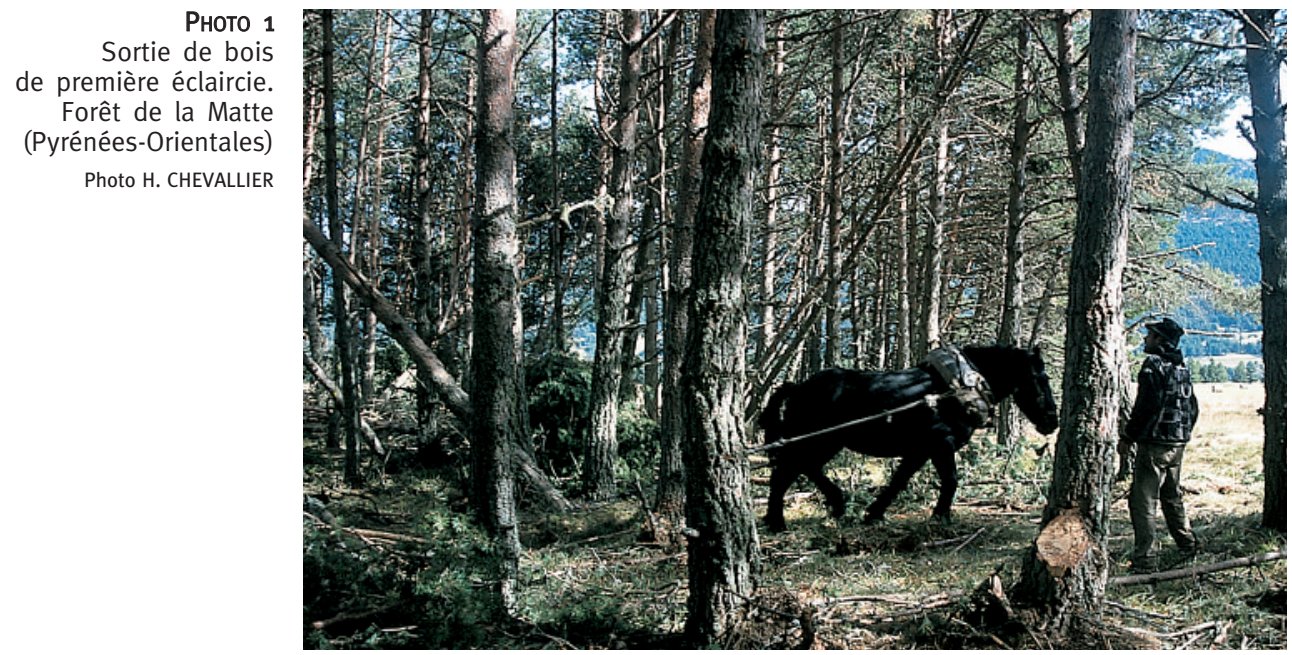

Plantés ou issus de régénérations naturelles, les peuplements ont pour caractéristique générale de n'avoir jamais été éclaircis, sauf exception ou simple nettoyage pour bois de chauffage. Les densités sont souvent fortes, jusqu'à 6000 tiges/ha et le rapport de la hauteur au diamètre (H/D) est généralement supérieur à 80 .

Selon le type de parcelles d'origine, les propositions d'actions varient (tableau III) :

TABLEAU III

Propositions d'actions selon le type de parcelle

\begin{tabular}{|l|l}
\hline $\begin{array}{l}\text { Pré colonisé par du Pin } \\
\text { sylvestre, en bouquets } \\
\text { ou isolés (type 1) }\end{array}$ & $\begin{array}{l}\text { - Activer ou assister la régénération naturelle, dépressage des bouquets } \\
\text { ou plantation } \\
\text { ou maintien des zones ouvertes par une reprise agricole } \\
\text { (coupe rase du bois existant: mobilisation de petits bois) }\end{array}$ \\
\hline $\begin{array}{l}\text { Peuplement naturel } \\
\text { de Pin sylvestre } \\
\text { ou plantation (type 2 ou 3) }\end{array}$ & $\begin{array}{l}\text { - Éclaircie sélective, élimination des brins sans avenir, } \\
\text { objectif : 900-1000 t/ha } \\
\text { - Mobilisation de bois de trituration et de petits sciages } \\
\text { ("sapin de Noël” avec l'Épicéa par exemple) } \\
\text { Conservation de bois morts, sur pied ou à terre }\end{array}$
\end{tabular}

En lien avec la filière bois-énergie développée sur le département des Pyrénées-Orientales, les produits de première éclaircie, habituellement laissés sur place, seront broyés et utilisés pour la production de plaquettes (utilisation pour les chaudières à bois).

Certains autres aspects complémentaires sont pris en compte selon les parcelles.

À proximité d'un ravin, il est proposé de conserver la zone en pré-bois et de concentrer les éclaircies sélectives sur le reste de la parcelle.

L'aspect paysager également doit être pris en compte notamment dans le cas de parcelles bordant la route.

Sur les parcelles ayant fait l'objet de précédentes coupes rases, la régénération peut avoir du mal à s'installer du fait d'un tapis herbacé trop dense. Un travail du sol est à envisager alors, et on propose de passer à une plantation en attachant un soin particulier au choix des essences. 
D’un point de vue paysager, trois zones ont été définies selon la vocation des parcelles : zones d'appel ou d'accueil, zones périphériques ou tampon, zones forestières. Les aménagements paysagers découlent directement de la vocation définie de tel ou tel secteur (tableau IV).

TABLEAU IV Aménagements paysagers selon la zone concernée

\begin{tabular}{|c|c|c|}
\hline Vocation de la zone & Objectif & Action à envisager \\
\hline \multirow[t]{2}{*}{$\begin{array}{l}\text { Zones d'accueil } \\
\text { ou d'aménagement }\end{array}$} & $\begin{array}{l}\text { Rendre attractive la } \\
\text { zone concernée }\end{array}$ & $\begin{array}{l}\text { - coupe sanitaire } \\
\text { - élagage } \\
\text { - débroussaillage } \\
\text { - broyage intégral des résidus d’élagage } \\
\text { et de débroussaillement }\end{array}$ \\
\hline & Équiper la zone & $\begin{array}{l}\text { - eau potable, bacs à ordures, tables, } \\
\text { signalisation, (re)calibrer la capacité d'accueil } \\
\text { (délimitation des places de parking) }\end{array}$ \\
\hline $\begin{array}{l}\text { Zones périphériques } \\
\text { ou zones tampon }\end{array}$ & $\begin{array}{l}\text { Améliorer l'aspect } \\
\text { visuel aux abords } \\
\text { des zones d'accueil }\end{array}$ & $\begin{array}{l}\text { - élagage pour une plus grande profondeur } \\
\text { de champ } \\
\text { - pas de débroussaillage (aucune utilité) }\end{array}$ \\
\hline Zones forestières & $\begin{array}{l}\text { Diminuer } \\
\text { l’hétérogénéité } \\
\text { paysagère }\end{array}$ & $\begin{array}{l}\text { - éclaircie, dépressage et nettoyage des zones } \\
\text { visibles depuis une route par exemple. }\end{array}$ \\
\hline
\end{tabular}

La composition avifaunistique est en relation directe avec le type de formation forestière majoritaire du secteur étudié. Dans les pinèdes de Pin sylvestre dominent les Mésanges, noire et huppée, et le Roitelet huppé. Cependant, la sylviculture joue un rôle tout de même important car elle façonne la structure et la physionomie des peuplements, facteurs essentiels de la distribution des oiseaux dans l'écosystème forestier.

Ce diagnostic avifaunistique a d'ores et déjà soulevé la question des vieux arbres, dont dépendent plusieurs espèces sur ce secteur, parmi lesquelles certaines sont d'importance patrimoniale remarquable ou en diminution sur cette région (Chouette de Tengmalm, Pic noir, Pigeon colombin). Le maintien des vieilles parcelles forestières ou d'arbres âgés s'avère indispensable pour la conservation et l'étude de ces espèces, notamment pour estimer leur probabilité de survie dans cette forêt exploitée.

Le diagnostic pastoral a fait notamment ressortir le besoin d'une meilleure utilisation des ressources fourragères, que ce soit pour limiter l'excès ou le défaut de pâturage. Une attention doit également être portée au calendrier de pâturage des différents lots, et ce pour différentes raisons :

- présence du Grand Tétras : passage tardif (à partir de la mi-août) des animaux d'élevage pour éviter le dérangement des nichées,

- régénération forestière : coupes et mise en défens temporaire.

Quel est l'intérêt d'une telle démarche ? Par une approche concertée et multiple, de nombreux aspects complémentaires affinent et améliorent le choix des actions. Il apparaît ainsi possible de "faire quelque chose" de la petite propriété privée sur ce territoire. Cela demande un effort important d'animation auprès des propriétaires mais, derrière, se profile l'espoir de pouvoir valoriser des parcelles forestières plus ou moins à l'abandon, qui manquent cruellement de suivi et de gestion. Cela se traduira ensuite en terme de paysage : une forêt gérée permet d'aborder les problèmes paysagers rencontrés sur les zones de forte dynamique forestière. 


\section{PORTER À CONNAISSANCE POUR POUVOIR DIALOGUER}

À l'occasion de ce travail de préfiguration sur la forêt de la Matte, les différentes parties prenantes ont vu l'intérêt d'élargir cette approche multivariée à l'ensemble du territoire. L'objectif est devenu alors l'élaboration d'un outil simple, à l'usage des gestionnaires, permettant d'intégrer les enjeux patrimoniaux dans la définition de pratiques forestières. Au-delà des professionnels, il s'agit de sensibiliser les propriétaires à ces questions d'actualité en leur proposant un guide pratique permettant d'aborder ces différentes questions :

- quelles stations, quelles potentialités ?

- quels habitats, quelles espèces, quels enjeux ? (photo 2, ci-contre).

- quelles pratiques, quelles orientations ?

Ce travail a soulevé quelques interrogations :

- d'ordre général sur l'application du document : difficultés d'échelle, précision recherchée, objectifs différents selon les approches, patrimoniale ou économique ;

- d'ordre écologique, d'après les constatations de terrain : difficultés du Pin sylvestre à se régénérer sous lui-même, quelle dynamique ? Homogénéité du Pin à crochets : estil toujours vraiment en station ? Quelles conclusions en tirer?

Рното 2

Paysage forestier du Conflent

Photo H. CHEVALLIER

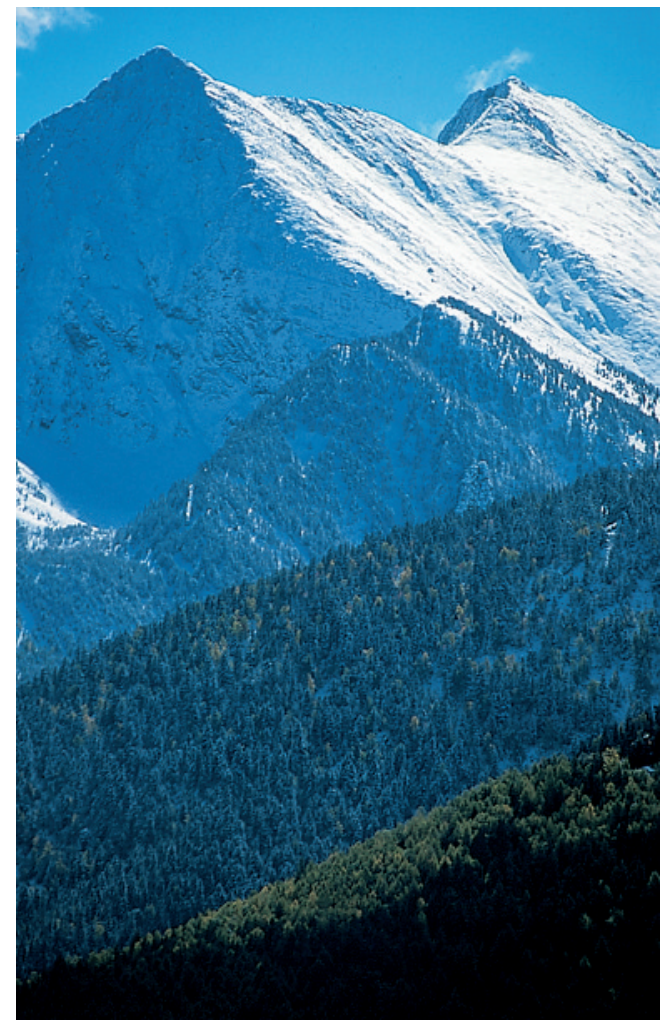

L'outil, sous forme de guide pratique, fournit les éléments de diagnostic qui permettront :

- l'identification des différentes formations forestières par une double approche station/habitat,

- l'amélioration des connaissances sur la dynamique des milieux forestiers sur un territoire où la forêt est jeune et l'influence des activités humaines très marquée,

- l'identification de précautions et conseils pour le choix des essences, les modes de gestion sylvicoles, pastoraux ou écologiques.

\section{PERSPECTIVES ET CONTINUATION}

Le projet de parc naturel régional des Pyrénées catalanes est l'occasion, sur un territoire complexe, de tenter une approche homogène et unifiée dans la méthode de description et d'identification, l'objectif étant ensuite d'avoir une référence travaillée en concertation et acceptée 
par tous les acteurs locaux, dans le but de faciliter l'élaboration de futures règles de gestion et de suivi.

Ces réflexions alimenteront la rédaction de la charte du futur parc naturel régional et pourraient servir de base de travail pour la mise en place d'éventuelles "chartes de territoires forestiers" à l'échelle d'un massif, applicables au niveau de la petite propriété privée.
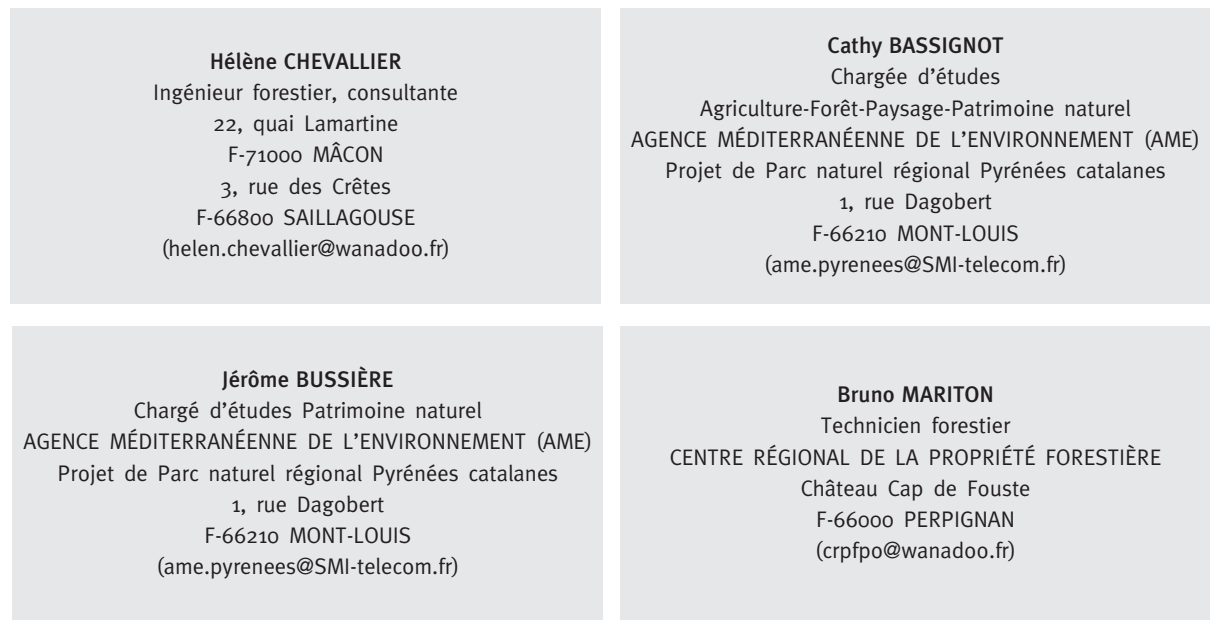

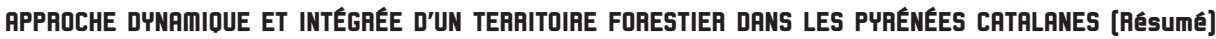

Patrimoines naturels forestiers et paysages sont au cœur des réflexions menées actuellement autour d'un projet de Parc naturel régional sur les Pyrénées catalanes. Dans ce contexte, gestionnaires forestiers, pastoralistes, agriculteurs, naturalistes et scientifiques se sont retrouvés autour de différents projets.

Deux grandes orientations actuelles : favoriser une approche concertée et multiple; proposer des outils simples permettant d'intégrer les enjeux patrimoniaux dans la définition de pratiques forestières.

Le projet de Parc naturel régional des Pyrénées catalanes est l'occasion sur un territoire complexe de tenter une approche homogène dans la méthode de description et d'identification. L'objectif étant ensuite d'avoir une référence travaillée en concertation et acceptée par tous les acteurs locaux, dans le but de faciliter l'élaboration de futures règles de gestion et de suivi.

\section{AN INTEGRATED DYNAMIC APPROACH TO A FOREST MASSIF IN THE CATRLAN PYAENEES [Abstract]}

Natural forest heritages and landscapes are a central concern of investigations relating to the Natural Regional Park project in the Catalan Pyrenees. Forest managers, range scientists, farmers, naturalists and researchers have come together to examine various plans.

The focus so far is on two major policies - fostering broad-based consultations, offering simple tools that incorporate heritage considerations into the definition of forest practices.

The plan for the Regional Natural Park in the Catalan Pyrenees is an opportunity to try to implement a consistent approach for the description and identification method of a complex geographic entity. The ultimate goal is to establish a benchmark that has been achieved through consultations and accepted by the various local stakeholders so as to pave the way for establishing future management and monitoring rules. 